\title{
Prominence fine structures in a magnetic equilibrium
}

\section{Lyman continuum in 2D configurations}

\author{
S. Gunár ${ }^{1,2,3}$, P. Heinzel ${ }^{1}$, and U. Anzer ${ }^{2}$ \\ 1 Astronomical Institute, Academy of Sciences of the Czech Republic, 25165 Ondřejov, Czech Republic \\ e-mail: [gunar;pheinzel] @asu.cas.cz \\ 2 Max-Planck-Institut für Astrophysik, Karl-Schwarzschild-Strasse 1, 85740 Garching, Germany \\ e-mail: ula@mpa-garching.mpg.de \\ 3 Astronomical Institute, Faculty of Mathematics and Physics, Charles University, Prague, Czech Republic
}

Received 29 July 2006 / Accepted 19 October 2006

\begin{abstract}
Aims. We discuss the behavior of the Lyman continuum profiles studied on the grid of 2D vertical-thread models for prominence fine structures.

Methods. Multilevel non-LTE transfer calculations for a 12-level plus continuum hydrogen model atom are used.

Results. Since the Lyman continuum is formed in regions with different temperatures for different orientations between the magnetic field direction and the line-of-sight, our Lyman continuum modeling, together with additional information from Lyman lines, represents a very useful tool for the determination of the thread structure.

Conclusions. A comparison between our theoretical Lyman continuum models between $800 \AA$ and $911 \AA$ with the observed values shows that such a modeling can give interesting new constraints on the temperature structure in prominence threads.
\end{abstract}

Key words. Sun: prominences - radiative transfer - line: profiles

\section{Introduction}

In the past ten years the observations by the SUMER UV-spectrograph (Wilhelm et al. 1995) on board SOHO (SOlar and Heliospheric Observatory) have produced a considerable amount of spectral data of hydrogen Lyman lines and of the continuum in prominences. Some of these data and their analysis can be found in Schmieder et al. (1998, 1999, 2003), Heinzel et al. (2001), and Gunár et al. (2006); see also a review of SOHO prominence observations by Patsourakos \& Vial (2002) and Heinzel et al. (2006). These Lyman line and continuum observations represent an important constraint for prominence modeling. To interpret them properly one has to use highly sophisticated models with complex non-LTE radiative transfer computations and include the prominence-corona transition region (PCTR). An earlier study by Fontenla et al. (1996) considered an ensemble of many thin vertical 1D slabs, each having its own PCTR. They found that their results are not entirely compatible with the available observations and therefore some magnetic shear will be required. More recently Labrosse \& Gouttebroze (2004) presented radiative transfer calculations of the He I triplet lines in a $1 \mathrm{D}$ vertical slab and also concluded that the presence of a PCTR will be required to match the observations. 2D models of the prominence fine structure were presented by Heinzel $\&$ Anzer (2001, hereafter referred to as Paper I). They consist of two-dimensional vertically infinite threads in magnetohydrostatic (MHS) equilibrium (for further details see also Heinzel $\&$ Anzer 2005). These models consistently solve the 2D radiative transfer with partial frequency redistribution in Lyman lines, together with the MHS equations. The importance of such twodimensional modeling for a proper interpretation of the SUMER spectra was suggested by Heinzel et al. (2001). A detailed analysis of the SUMER spectra of two quiescent prominences shows a strong unreversed emission detected in Lyman lines higher than $\mathrm{L} \alpha$ in one case. The other prominence with a similar brightness in the Lyman spectra exhibits all lines strongly reversed. This can be explained by a different orientation of the magnetic field with respect to the line-of-sight. Thus the prominence threads are seen from different sides with the different shape of the PCTR. This idea is further corroborated by Heinzel et al. (2005, hereafter referred to as Paper II), where we have taken an important step towards the understanding of the behavior of the Lyman spectrum formation. While Paper II concentrated on the analysis of the Lyman line formation and no discussion of the Lyman continuum behavior has been done there, the purpose of this paper is to model the Lyman continuum. Section 2 of this paper describes our 2D modeling and Sect. 3 presents the discussion and gives our conclusions.

\section{2D modeling of the Lyman continuum}

The temperature structure of fine structure threads is characterized by a PCTR with a steep gradient of the temperature across the magnetic field lines and a shallower increase of the temperature along the field lines. Empirical temperature profiles of these threads were first used by Heinzel \& Anzer (2001), who assumed a simple analytical description with

$T(m, y)=T_{\text {cen }}(y)+\left[T_{\text {tr }}-T_{\text {cen }}(y)\right]\left\{1-4 \frac{m}{M(y)}\left[1-\frac{m}{M(y)}\right]\right\}^{\gamma_{1}}$

where $T_{\text {tr }}$ represents the temperature at the boundary (for the hydrogen plasma was set to the $50000 \mathrm{~K}$ ) and the exponent $\gamma_{1}$ determines the gradient of the temperature along the field lines 
( $x$-direction). Column-mass scale $m$ is parallel to the $x$-direction with a simple relation through the plasma density. The temperature in the mid-plane (i.e., at $x=0$ ) is given by

$T_{\text {cen }}(y)=T_{\text {tr }}-\left(T_{\text {tr }}-T_{0}\right)\left(1-\left|\frac{y}{\delta}\right|^{\gamma_{2}}\right), \quad$ for $\quad|y| \leq \delta$,

where $T_{0}$ is the (minimum) central temperature (here we take $8000 \mathrm{~K}), 2 \delta$ represents the width of the thread perpendicular to the field lines (the width for our model is $1000 \mathrm{~km}$ ), and the exponent $\gamma_{2}$ represents the steep temperature gradient across the field lines. The $2 \mathrm{D}$ temperature structure is then fully determined by the exponents $\gamma_{1}$ and $\gamma_{2}$. The density structure of the prominence thread was calculated for MHS equilibria in our Papers I and II. In the present paper we take these results into account. The magnetic field lines projected onto the $(m, y)$-plane are parallel to the $m$-direction.

Concerning limitations of our model: our 2D modeling of the prominence fine structure including the radiative transfer represents the most advanced approach. There are no such 3D models yet. However, we are about to work on a generalization of our model to 3D. We do not consider magnetic shear because our threads are always along the magnetic field independent of the fact that the field can be sheared with respect to the prominence/filament axis. Plasma flows in general do not have a strong influence on the Lyman continuum profiles (they are not affected by Doppler shift).

\section{Effect of the different temperature structure}

To show the influence of different temperature structure on the Lyman continuum we use the models $\boldsymbol{A}_{1}\left(\gamma_{1}=5\right)$ and $\boldsymbol{C}_{1}\left(\gamma_{1}=\right.$ 10) of our Paper II. Model $\boldsymbol{B}_{1}$ differs from $\boldsymbol{C}_{1}$ only in the value of the parameter $\gamma_{2}$. Since in these models the PCTR slab across the field lines is very thin, the Lyman continuum is formed in regions deeper in the thread and is not directly influenced by $\gamma_{2}$. Minor effects of $\gamma_{2}$ on the Lyman continuum profile are due to its influence on the global shape of the temperature structure. That results in similar Lyman continuum profiles for models $\boldsymbol{B}_{1}$ and $\boldsymbol{C}_{1}$. The other models of our grid from Paper II $\left(\boldsymbol{A}_{2}, \boldsymbol{A}_{3}\right.$, etc. $)$ show the same behavior as $\boldsymbol{A}_{1}$ and $\boldsymbol{C}_{1}$, but with smaller Lyman continuum intensities.

The parameter $\gamma_{1}$ prescribes the PCTR area along the field lines characterized by a shallower gradient of the temperature. The formation depth of the Lyman continuum is inside this region and thus the exponent $\gamma_{1}$ has a significant effect on the Lyman continuum profile. The basic parameters of the models $\boldsymbol{A}_{1}\left(\gamma_{1}=5\right)$ and $\boldsymbol{C}_{1}\left(\gamma_{1}=10\right)$ are: $M_{0}=2 \times 10^{-4} \mathrm{~g} \mathrm{~cm}^{-2}$; $B_{x}(0)=8.4$ Gauss; $\gamma_{2}=30$, where $M_{0}$ is the maximum column density along the central field line and $B_{x}(0)$ is the horizontal field in the middle of the thread. The only difference is thus in the parameter $\gamma_{1}$.

The Lyman continuum profiles for these two models are shown in Fig. 1. Here the full lines represent averaged profiles over the width of the thread (profiles seen along the field lines), the dashed lines are mean profiles over the whole length of the thread (seen across the field lines). Double-arrowed lines belong to model $\boldsymbol{C}_{1}$. The main difference between the Lyman continuum profiles for models $\boldsymbol{A}_{1}$ and $\boldsymbol{C}_{1}$ lies in the value of the specific intensity at each wavelength. As is shown in Fig. 1, the specific intensity for model $\boldsymbol{A}_{1}$ is almost twice as high as for model $\boldsymbol{C}_{1}$, while the difference between the two directions of view is almost one order of magnitude. For a detailed interpretation of these calculations we further use the contribution functions for a set of wavelengths of the Lyman continuum.

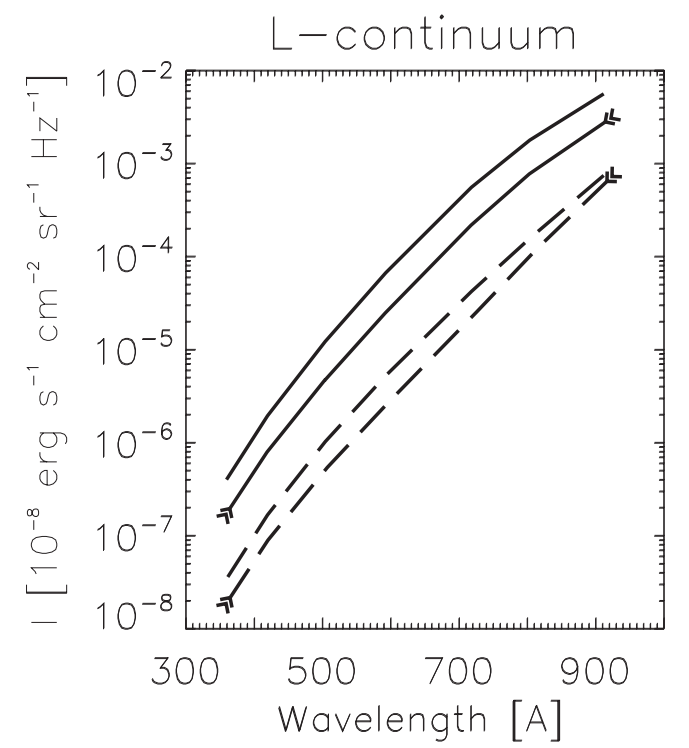

Fig. 1. Lyman continuum for models $\boldsymbol{A}_{1}$ and $\boldsymbol{C}_{1}$. Full lines represent averaged profiles over the width of the thread, dashed lines are mean profiles over the whole length of the thread. Double-arrowed lines belong to the model $C_{1}$.

The description of the contribution-function computation was given in Paper II. We show panels of plots of contribution functions for both models and certain wavelengths with overplotted contours of temperature and density. The bold dashed contour represents the level of the optical depth of $\tau=1$ at the selected wavelength. Each plot shows the spatial variation of the contribution to the specific intensity for a given wavelength as a function of the geometrical position. The white regions represent major contributions, the black ones are regions with minor contribution. The panel plots for given wavelengths (Figs. 2-4) are organized as follows: the first four plots are for model $\boldsymbol{A}_{1}$, the second four for model $\boldsymbol{C}_{1}$. The upper pair of plots represents viewing along the field lines (from the left side in our plots, marked as $x$-direction according the geometry of the thread), the lower pair represents viewing across the field lines (from the bottom in our plots, marked as $y$-direction). On the left-hand side are the contribution functions over-plotted by iso-lines of the temperature (contours represent 9000, 10000, 12000, $15000,20000,30000$, and $40000 \mathrm{~K}$ starting at the center of the thread). On the right-hand side are the iso-lines of the density for $0.25,0.5,1.0,1.5,2.0$, and $2.5 \times 10^{-13} \mathrm{~g} \mathrm{~cm}^{-3}$ starting at the borders of the thread. Note that these figures are not drawn to the true scale (they are squeezed in the $x$-direction). We shall now discuss the results of our model calculations (shown in Fig. 1) for wavelengths that lie between the head of the Lyman continuum at $911 \AA$ and the head of the He I continuum at $504 \AA$.

\section{$911 \AA$}

When viewed along the field lines (from the left side) the maximum of the contribution function for model $\boldsymbol{A}_{1}$ is in the region with the temperature between 12000 and $17000 \mathrm{~K}$ (Fig. 2). The specific intensity of model $\boldsymbol{C}_{1}$ is formed at slightly lower temperatures (10000 to $15000 \mathrm{~K}$ ) and also at lower densities. Therefore the value of the specific intensity at the wavelength of $911 \AA$ for model $\boldsymbol{A}_{1}$ is two times higher $\left(6.0 \times 10^{-10} \mathrm{erg} \mathrm{s}^{-1} \mathrm{~cm}^{-2} \mathrm{sr}^{-1} \mathrm{~Hz}^{-1}\right)$ than for model $\boldsymbol{C}_{1}\left(3.0 \times 10^{-10} \mathrm{erg} \mathrm{s}^{-1} \mathrm{~cm}^{-2} \mathrm{sr}^{-1} \mathrm{~Hz}^{-1}\right)$ (see also Fig. 1). In the direction of view across the field lines (from the bottom) 

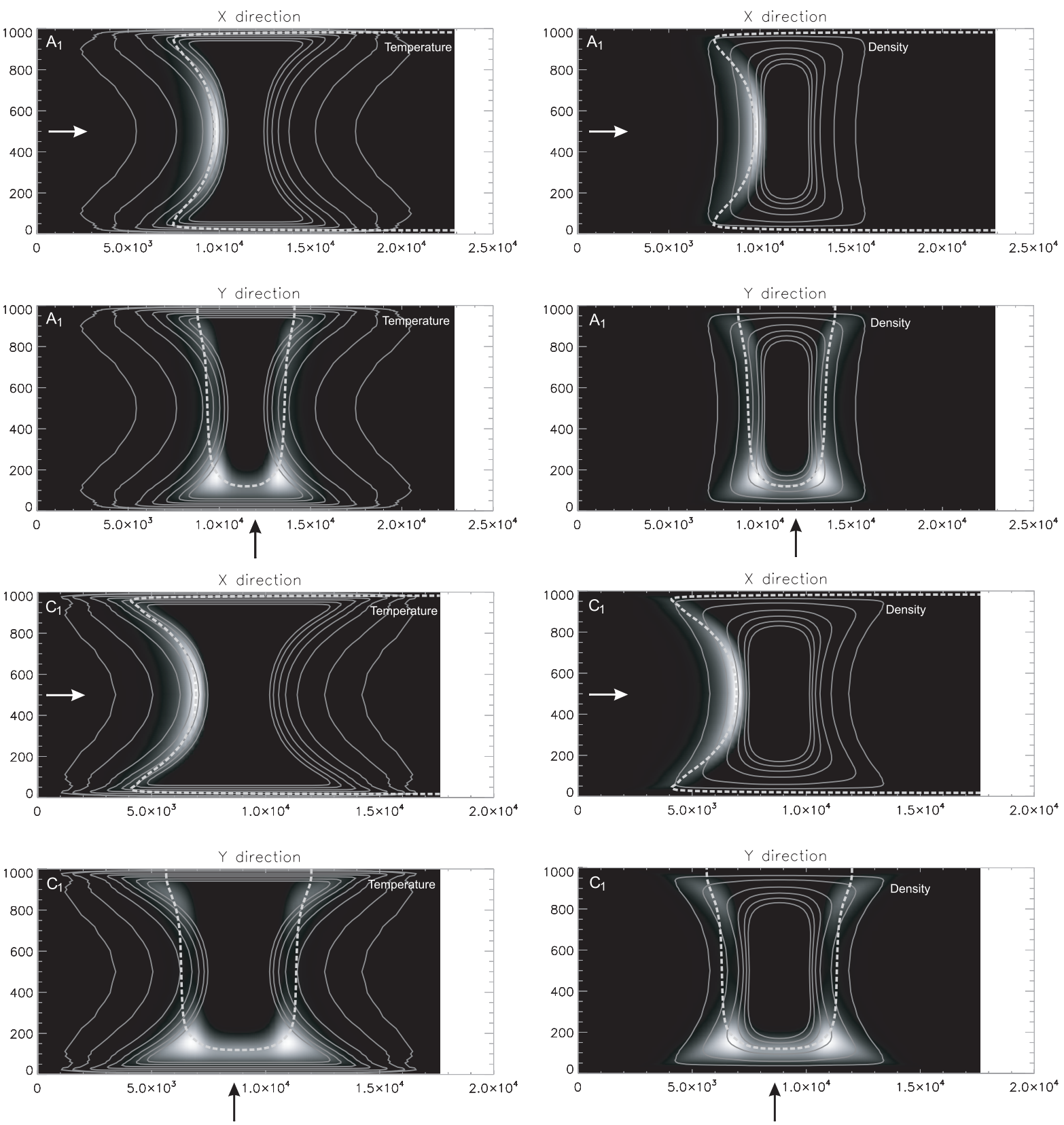

Fig. 2. Contribution function for Lyman continuum at the wavelength $911 \AA$ for models $\boldsymbol{A}_{1}$ (the first four plots) and model $\boldsymbol{C}_{1}$ (the second four plots). The upper pair of the plots for each model represents the viewing along the field lines (from the left side), the lower pair represents the viewing across the field lines (from the bottom). On the left-hand side iso-contours of the temperature $(9000,10000,12000,15000,20000$, 30000 , and $40000 \mathrm{~K})$ and on the right-hand side iso-contours of the density $\left(0.25,0.5,1.0,1.5,2.0\right.$, and $\left.2.5 \times 10^{-13} \mathrm{~g} \mathrm{~cm}^{-3}\right)$ are shown. The bold dashed line represents the contour of $\tau(911 \AA)=1$.

the maximum of the contribution function is in the central region with minimal temperature for both models; however, for model $\boldsymbol{A}_{1}$ this area has a higher density. The value of the specific intensity varies along the length of the thread. In the central cool part it is almost the same for both models, while the maximum of the intensity is twice as high for model $\boldsymbol{A}_{1}$ as for model $\boldsymbol{C}_{1}$. The areas with the major contribution correspond to the $\tau=1$ contour. We also have similar results for the wavelength of $803 \AA$.

\section{$717 \AA$}

For the direction of view along the field lines the specific intensity comes from the region with temperatures from 10000 up to $20000 \mathrm{~K}$ for model $\boldsymbol{A}_{1}$ and up to $18000 \mathrm{~K}$ for model $\boldsymbol{C}_{1}$ with slightly higher density for model $\boldsymbol{A}_{1}$ (Fig. 3). The specific intensity is again much higher for model $\boldsymbol{A}_{1}$ (Fig. 1). Viewed across the field lines the place of formation for model $\boldsymbol{A}_{1}$ is shifted to the same area as for the direction of view along the field. However, across the field lines we see two symmetrical areas of major contribution. The specific intensity of model $\boldsymbol{C}_{1}$ is partially formed in the central cool part of the thread and partially in the same region as viewed along the field lines. The value of the specific intensity is higher for model $\boldsymbol{A}_{1}$ everywhere along the length of the thread.

\section{$504 \AA$}

For both directions of view the specific intensity is formed at higher temperatures from 15000 to more than $30000 \mathrm{~K}$, but 

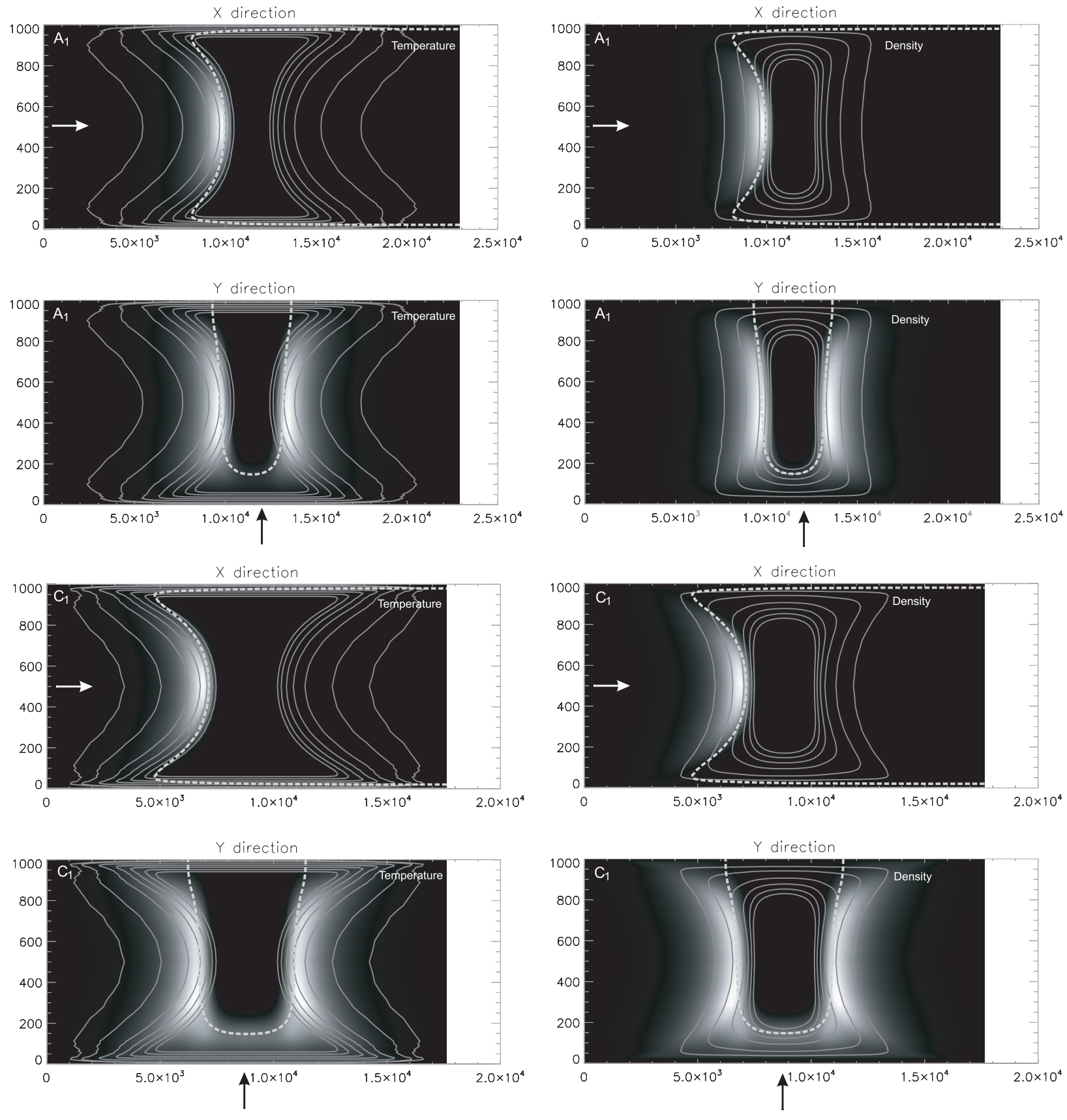

Fig. 3. Same as in Fig. 2 but at the wavelength $717 \AA$.

the density is higher for model $\boldsymbol{A}_{1}$ (Fig. 4). Therefore the intensity for model $\boldsymbol{A}_{1}$ is higher than for model $\boldsymbol{C}_{1}$ in both directions of view. All contributions come from regions lying in front of the $\tau=1$ contour because of the insufficient temperature at lower wavelengths of the Lyman continuum in the $\tau=1$ region. Similar results (assuming pure hydrogen) were also obtained for wavelengths below $504 \AA$.

The parameter $\gamma_{1}$ prescribes the gradient of the temperature along the field lines. With a lower value of $\gamma_{1}$ we have a smoother increase of temperature from the minimal value $T_{0}=8000 \mathrm{~K}$ to the temperature at the boundary $T_{\mathrm{tr}}=50000 \mathrm{~K}$. This results in higher temperatures and densities in areas where the Lyman continuum for a certain wavelength is formed and thus to higher values of the specific intensity for models with lower values of the exponent $\gamma_{1}$.

\section{Effect of the fine structure orientation}

The effect of the fine-structure thread orientation with respect to the line-of-sight is given by the shape of the temperature structure, which is characterized by the PCTR as outline in our Sect. 2. To show the difference between Lyman continuum profiles seen along and across the field lines we use model $\boldsymbol{C}_{1}$. For a detailed analysis the wavelengths of $911 \AA$ and $803 \AA$ are useful since their formation region when seen across the field lines is different from the one viewed along the field in comparison to the lower wavelengths.

In the case that the line-of-sight is oriented along the field lines, the Lyman continuum in the range $911 \AA$ to $803 \AA$ is formed in the PCTR with the temperature gradient described by $\gamma_{1}$ in the area with temperatures from 10000 to $15000 \mathrm{~K}$. When seen across the field lines the place of the formation is in 

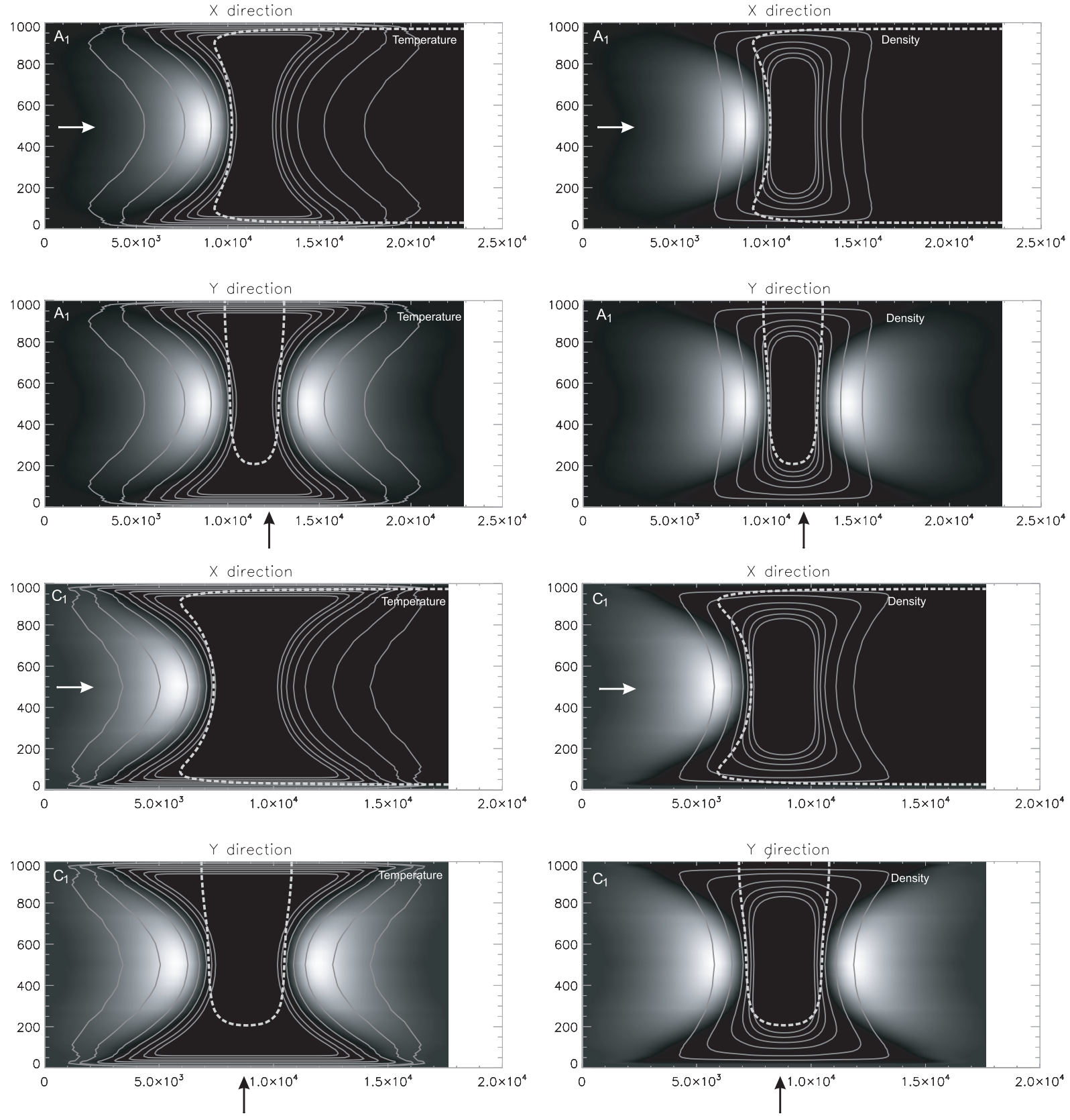

Fig. 4. Same as in Fig. 2 but at the wavelength $504 \AA$.

the central (coolest) part of the thread. The contribution function for this direction of view has two peaks with an area of minor contribution between them (Fig. 2). Also according to the contribution function, the specific intensity has two peaks at the same position (Fig. 5). The position of the peaks of the specific intensity can be explained by the location of the $\tau=1$ line. The contribution function follows the $\tau=1$ line and has a maximum at the places where the $\tau=1$ line is curved inwards. Thus we see there a bigger contributing region than in the center of the thread.

In the direction of view across the field lines the Lyman continuum is formed at temperatures significantly lower than those seen along the field lines. This is shown also in Fig. 6 where the bold line represents the averaged profile over the width of the thread (profile seen along the field lines), which is formed at a higher temperature than the continuum seen across the field lines. The other lines represent Lyman continuum profiles in

different positions along the length of the thread from the center of the thread to the position with the maximum intensity (see Fig. 5). The specific intensity is drawn in logarithmic scale.

\section{Discussion and conclusions}

This investigation shows that the prominence fine structure can have a very strong influence on the shape of the observed Lyman continuum. There are several aspects that one has to take into account for the interpretation of such spectra. The presence of the PCTR is very important for the emission of the Lyman continuum. A direct comparison of Figs. 2 to 4 shows that for the wavelengths around $911 \AA$ the contribution mainly comes from the innermost parts of the PCTR and also from parts of the cool prominence interior. The location of the emission also strongly depends on the viewing direction with respect to the magnetic field. At 911 and $803 \AA$, the emission is centered around the 


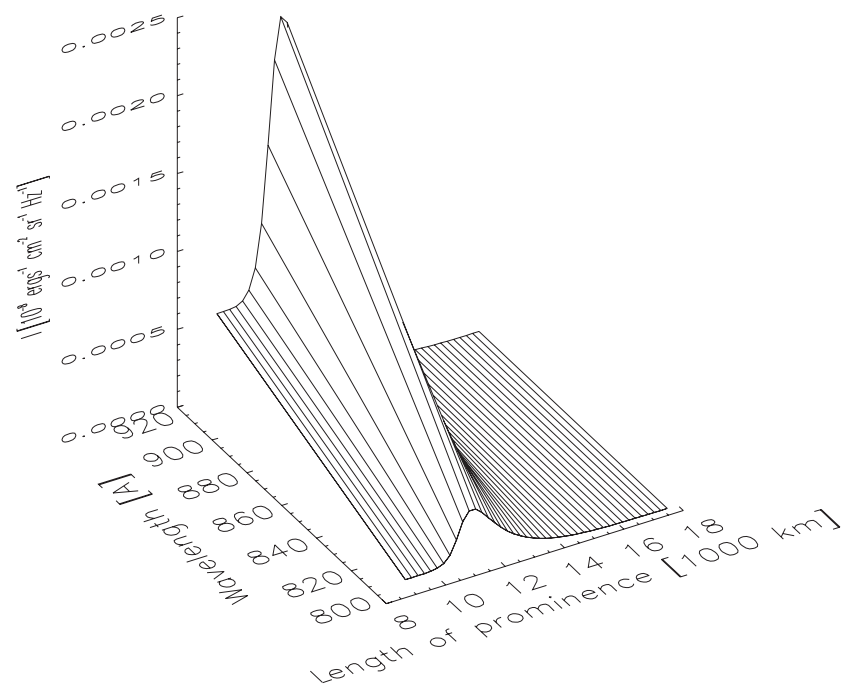

Fig. 5. Lyman continuum intensity variation along the thread for model $\boldsymbol{C}_{1}$. The plot represents one half of the thread from its center.

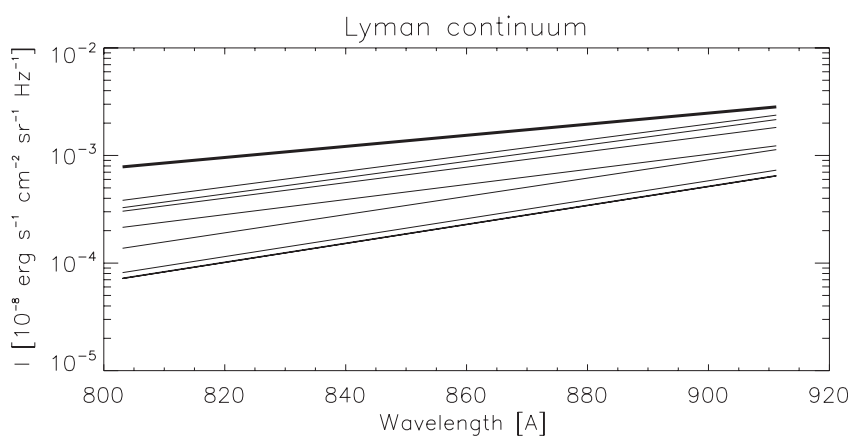

Fig. 6. Lyman continuum for model $\boldsymbol{C}_{1}$. The bold line represents an averaged profile seen along the field lines. Other lines belong to different positions along the length of the thread (seen across the field) from the center of the thread (the lowest lines) to the peak (the highest lines).

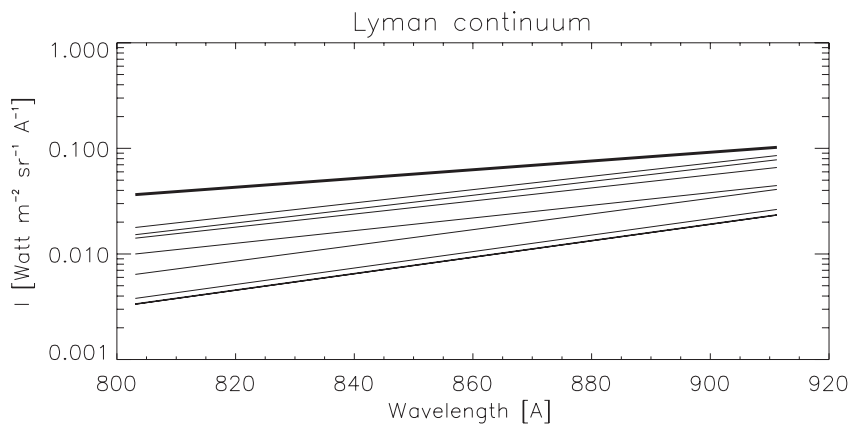

Fig. 7. Same plot as in Fig. 6, but the intensity is expressed in Watt $\mathrm{m}^{-2} \mathrm{sr}^{-1} \AA^{-1}$ for a better comparison with Parenti et al. (2005).

$\tau=1$ line. At $718 \AA$, and even more pronounced at $504 \AA$, the emission basically comes from hotter parts of the PCTR and the region is spatially much more extended. In these cases it comes from regions in front of the $\tau=1$ line (i.e., optically thin regions). The emergent spectra vary strongly as one moves along the threads (Fig. 5). This effect will make any temperature determination on this basis rather uncertain unless we can reach a very high spatial resolution of individual threads.

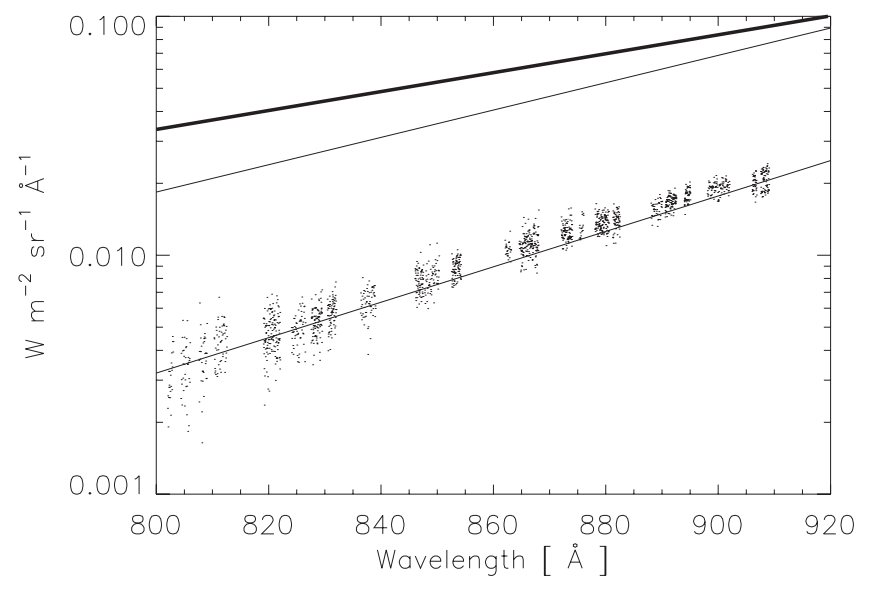

Fig. 8. Intensity of the Lyman continuum (corrected) from part A_1 of the prominence discussed by Parenti et al. (2005). The bold line represents an averaged profile of model $\boldsymbol{C}_{1}$ seen along the field lines. Other lines belong to different positions of model $\boldsymbol{C}_{1}$ along the length of the thread (seen across the field).

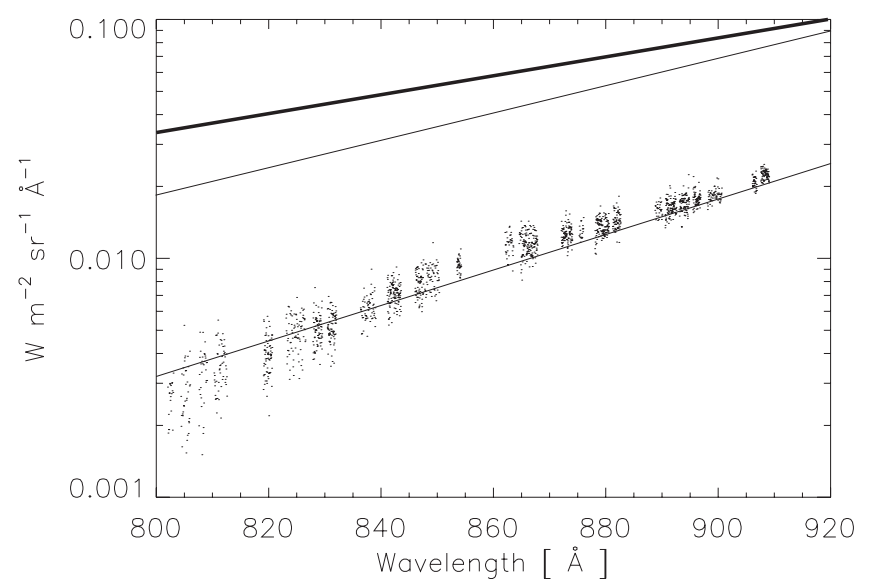

Fig. 9. Same as in Fig. 8, but for part A_2 of the prominence.

The situation is somewhat clearer if one only considers the part of the spectrum between $800 \AA$ and $911 \AA$, as was done in Parenti et al. (2005). Then the main contributions over this entire spectral range come from approximately the same locations in space. In such a case, it is possible to interpret this spectrum in terms of a mean temperature of the contributing region. But the contributing regions still depend strongly on the viewing direction. From Fig. 2 one sees that the contribution comes from a region with the temperature between 10000 to $15000 \mathrm{~K}$ when viewed along the field lines (model $\boldsymbol{C}_{1}$ ) and from regions with the temperature slightly higher than $8000 \mathrm{~K}$ when looking perpendicular to the field lines (see Fig. 7). In the first case one looks at the innermost parts of the PCTR, in the second mainly at the cooler prominence body. Therefore the temperatures derived by making spectral fits to the observations will be strongly dependent on the fine structure and the viewing angle. But in all cases the temperature derived on this basis will lead to upper limits for the true central prominence temperature.

All profiles seen across the field lines (Fig. 7) come from regions with similar temperatures, the only difference is in the $b$-factors (i.e., non-LTE departure coefficients for the ground level of the hydrogen). The lowest profiles with the highest values of the $b$-factors correspond to the very central part of the thread. These profiles represent satisfactory fits to the data from 
part A_1 of the prominence discussed by Parenti et al. (2005) (Fig. 8). Their derived temperature $(8300 \mathrm{~K})$ is slightly higher than the central temperature that we used in our models. For part A_2 (Fig. 9) they obtained a temperature of $7600 \mathrm{~K}$, which is lower than the minimum temperature of our set of models. Note that Parenti et al. (2005) derived the color temperature from spectra in Figs. 8 and 9 and then assigned it to the kinetic temperature. This is based on previous results of Gouttebroze et al. (1993).

Our computations show that temperatures derived by using the color temperature can be considered as upper limits for the central prominence temperature. This is due to the variation of the $b$-factors along the wavelength, which allows us to make same fit to the spectra in Figs. 8 and 9 with lower temperature. The temperatures determined by Parenti et al. (2005) ranging from 7600 to $8300 \mathrm{~K}$ are actually not in conflict with possibly lower values in the central parts.

This paper shows that the Lyman continuum spectrum between $800 \AA$ and $911 \AA$ alone does not give a unique answer about the plasma parameters in prominences. Therefore it will be desirable to analyze simultaneous measurements of this continuum (in a broader wavelength range) together with the detailed spectra of different Lyman lines. This can give more rigorous constraints on the models and therefore will allow one to obtain more reliable estimates of the physical conditions in quiescent prominences.
Acknowledgements. S.G. and P.H. acknowledge the support from the MPA Garching; U.A. thanks for support from the Ondřejov Observatory. This work was done during S.G.'s stay at the MPA Garching supported by the EARA-EST programme and in the frame of grant No. IAA 3003203 of the Grant Agency of the Academy of Sciences of the Czech Republic and ESA-PECS project No. 98030. We thank S. Parenti for providing us with Figs. 8 and 9. SOHO is a space mission of international cooperation between ESA and NASA.

\section{References}

Anzer, U., \& Heinzel, P. 1999, A\&A, 349, 974

Fontenla, J. M., Rovira, M., Vial, J.-C., \& Gouttebroze, P. 1996, ApJ, 466, 496 Gouttebroze, P., Heinzel, P., \& Vial, J.-C. 1993, A\&AS, 99, 513

Gunár, S., Teriaca, L., Heinzel, P., \& Schuehle, U. 2006, in Proc. SOHO 17 Conf., ESA-SP, in press

Heinzel, P., \& Anzer, U. 2001, A\&A, 375, 1082 (Paper I)

Heinzel, P., \& Anzer, U. 2005, in Solar Magnetic Phenomena, ed. A. Hanslmeier, A. Veronig, \& M. Messerotti, Astron. Astrophys. Space Sci. Lib., 320 (Dordrecht: Springer), 115

Heinzel, P., Anzer, U., \& Gunár, S. 2005, A\&A, 442, 331 (Paper II)

Heinzel, P., Schmieder, B., \& Vial, J. C. 2006, in Proc. SOHO 17 Conf., ESA-SP, in press

Heinzel, P., Schmieder, B., Vial, J. C., \& Kotrč, P. 2001, A\&A, 370, 281

Labrosse, N., \& Gouttebroze, P. 2004, ApJ, 617, 614

Parenti, S., Lemaire, P., \& Vial, J.-C. 2005, A\&A, 443, 685

Patsourakos, S., \& Vial, J.-C. 2002, Sol. Phys., 208, 253

Schmieder, B., Heinzel, P., Kucera, T., \& Vial, J.-C. 1998, Sol. Phys., 181, 309

Schmieder, B., Heinzel, P., Vial, J.-C., \& Rudawy, P. 1999, Sol. Phys., 189, 109

Schmieder, B., Tziotziou, K., \& Heinzel, P. 2003, A\&A, 401, 361

Wilhelm, K., Lemaire, P., Curdt, W., et al. 1995, Sol. Phys., 170, 75 